ХУКАЛЕНКО Юлия Сергеевна,

канд. филол. наук, научный сотрудник Центра НТИ по нейротехнологиям, технологиям виртуальной и дополненной реальностей на базе ДВФУ (г. Владивосток).

Электронная почта: khukalenko.ius@dvfu.ru

\title{
Обучение иностранным языкам (на примере английского) с помощью технологии виртуальной реальности: обзор основных разработок
}

УДК 81.33

виртуальная

реальность,

информаи,ионные

технологии,

образовательные

технологии,

английский язык
Для изитирования:

Хукаленко Ю. С. Обучение иностранным языкам (на примере английского) с помощью технологии виртуальной реальности: обзор основных разработок // Известия Восточного института. 2021. № 2. C. $118-128$. DOI https://doi.org/10.24866/2542$1611 / 2021-2 / 118-128$
DOI https://doi.org/10.24866/2542-1611/2021-2/118-128

В данной работе представлен анализ инновационных решений с использованием виртуальной реальности для практики иностранных языков на примере английского языка. Выделены два типа программного обеспечения, применимых в рамках учебного процесса в школах и вузах: специализированные и смежные разработки. Результаты анализа демонстрируют, что виртуальная реальность как образовательная технология обладает потенциалом, однако всё ещё находится на начальном этапе своего развития, а существующие приложения нуждаются в методических доработках и дополнительных исследованиях эффективности.

\section{Введение}

В настоящий момент количество продуктов для обучения и практики иностранных языков в виртуальной реальности относительно невелико. Список научных исследований, посвящённых этой теме, также ограничивается небольшим количеством публикаций. Тем не менее в существующих работах говорится о плюсах виртуальной образовательной среды, таких как наглядность, вовлеченность, фокусировка, эффект присутствия, сверхстимуляция органов чувств, безопасность и многие другие $[1 ; 4 ; 7 ; 9 ; 10 ; 12]$. Разумеется, как любая новая технология, виртуальная реальность должна быть изучена гораздо полнее, и в ходе исследований должны быть выявлены её достоинства и недостатки по сравнению с другими инструментами. Однако потенциал технологии для образования не вызывает сомнений.

Оставляя терминологическую дискуссию за пределами данной работы, оговоримся, что в обзор попали лишь те приложения, которые предполагают использование шлема виртуальной реальности, поскольку для изучения языков принципиальным оказывается максимальный эффект присутствия [7]. Считается, что при экранном взаимодействии (так называемый desktop VR) иммерсивность снижается [5; 11].

Bсе приложения и платформы, на которых можно изучать иностранный язык, разделяются на две большие категории, которые мы назовём специализированными и смежными средствами. Под специализированными решениями мы будем понимать разработки, главной целью которых является непосредственно изучение языков, в то время как на смежных приложениях изучение языка является дополнительной возможностью. 


\section{1 Специализированные разработки}

\subsection{MondlyVR [14]}

MondlyVR от ATi Studios - это обучающее приложение виртуальной реальности, разработанное специально для изучения языков. Суть приложения в том, что учащийся «переносится» в виртуальную среду, где он или она может взаимодействовать с виртуальными персонажами посредством технологии распознавания речи.

Сразу после запуска программы учащийся указывает свой родной язык и выбирает один из тридцати языков для обучения (строго говоря, их там двадцать девять, т. к. британский и американский варианты английского даются как разные языки). Затем на выбор пользователю предоставляется две опции. Первая: можно выбрать коммуникативную ситуацию для практики речи, а именно, отработки определенного диалога и простых фраз. Таких ситуаций всего четыре: поезд, такси, стойка регистрации отеля и ресторан. Вторая опция - лексические мини-уроки по таким темам, как животные, овощи, фрукты, космос.

Несмотря на упрощение графических элементов (низкополигональная графика, примитивная анимация), дизайн не вызывает нареканий и способствует ощущению присутствия. Однако это ощущение исчезает при попытке речевого взаимодействия с персонажами. Вопервых, при обращении персонажа к учащемуся, последний может выбирать только из вариантов, предложенных программой. Эти варианты представляют собой достаточно громоздкий элемент интерфейса, который также негативно влияет на восприятие ситуации как реалистичной. Во-вторых, для записи голоса нужно навести указатель контроллера на элемент интерфейса, изображающего микрофон и нажать триггер, что делает речевое взаимодействие неспонтанным, а диалог - затянутым. И последнее, учащийся не может задавать вопросов собеседнику сам: инициатива в диалоге всегда принадлежит искусственному интеллекту.

Что касается лексических уроков, то в этой части программа проработана слабо. Используется технология speech-to-text, которая, несмотря на дешевизну, является неадекватной для целей обучения языку. Неправильные интонации, грубые грамматические ошибки («Я буду остаться в отеле до пятницы»), неузуальные употребления лексики и прочее делают робота не просто неэффективным, а скорее, вредным тренажером.

Таким образом, исключительно при условии улучшения и исправления ошибок, MondlyVR может быть встроен в образовательный процесс или рекомендован как дополнительный инструмент обучения. Такое приложение будет особенно полезно для учащихся с уровнем A2 (pre-intermediate) для практики элементарных диалогов, а также, возможно, для учащихся, испытывающих коммуникативные трудности, широко известные как «языковой барьер».

\subsection{Virtual Speech [15]}

В отличие от предыдущего приложения, виртуальная реальность на образовательных курсах от Virtual Speech (VirtualSpeech Ltd.) представляет собой дополнительный элемент в комплексной системе обучения. Так, дистанционный курс English for Business (английский для бизнеса) включает два компонента. Первый - стандартный онлайн курс с дизайном платформы FutureLearn (популярная образовательная платформа, на которой в основном размещаются массовые онлайн курсы) с видеолекциями, статьями для чтения, тестами и кейсами. Второй и не менее важный компонент курса - практика: 
полученные теоретические знания предлагается применить в виртуальной реальности.

Курс включает сорок девять уроков и шесть VR-сценариев. Рассмотрим, как это работает на примере темы «бизнес нетворкинг». После видеолекции и чтения дополнительной литературы, учащемуся предлагается применить полученные знания на практике. Надев шлем, учащийся оказывается на типичном профессиональном мероприятии, где он или она может поговорить последовательно с каждым из коллег. К сожалению, ощущение погруженности в коммуникативную ситуацию пропадает из-за неинтерактивности персонажей: они лишь проговаривают записанную аудиозапись, разделенную паузами, во время которых может говорить учащийся. При этом содержание речи учащегося программой игнорируется. Чтобы собеседник продолжил разговор после паузы, нужно нажимать на специальную кнопку интерфейса.

После завершения такого «диалога» система оценивает степень поддержки визуального контакта и предлагает мини-тест по услышанному материалу в форме выбора правильного варианта ответа. Например, нужно ответить, откуда собеседник, в какой компании работает, почему решил посетить данное событие и т. д. Такое упражнение не только тренирует навыки восприятия на слух звучащей иностранной речи, но также развивает внимательность и память, необходимые в профессиональном общении.

Отдельно отметим, что неинтерактивность персонажей не принципиальна в других сценариях, например, на репетиции публичного выступления. Предварительно загрузив свою презентацию, учащийся должен говорить достаточно продолжительное время перед виртуальной аудиторией из девяноста человек, стараясь не отвлекаться на тех, кто двигается, записывает или пьёт кофе. Полезной функцией является возможность сохранить аудиофайл своего выступления и впоследствии проанализировать его, либо поделиться с коллегами или преподавателем.

С появлением курсов, подобных описанному, можно с осторожностью говорить о новом типе смешанного обучения (blended learning), где вместо очного компонента выступает компонент виртуальной реальности. Вопрос об эффективности дистанционных автономных методов обучения иностранным языкам всё ещё остаётся открытым [6; 8], но инструмент виртуальной реальности, правильно встроенный в систему языкового онлайн курса, может послужит серьезным подспорьем тем учащимся, кто предпочитает осваивать языки самостоятельно онлайн.

\subsection{VARVARA [13]}

VARVARA - разработанная методистами Центра НТИ по нейротехнологиям, технологиям виртуальной и дополненной реальности ДВФУ серия курсов и сценариев-модулей для изучения и практики английского языка на запатентованной платформе ООО «ВиАр Суперсоник». Приложение разработано для учеников, находящихся на начальном этапе изучения английского языка (A1-A2 по общеевропейской системе оценки уровня владения иностранным языком) в возрасте от 10-11 лет. Программное обеспечение VARVARA направлено на решение сразу нескольких задач учителя: разговорная прак- 
тика иностранного языка в рамках урока, преодоление «языкового барьера» и боязни сделать ошибку, создание условий для повышения интереса к изучению английского. VARVARA соответствует требованиям ФГОС и содержанию учебного предмета «Иностранный язык (английский)».

В соответствии с концепцией разработчиков, диалоговый тренажёр не призван заменить учебник или учителя на уроке, а должен использоваться в качестве дополнения к традиционному уроку иностранного языка в школе. VR-сценарии сопровождаются руководством пользователя и методическими материалами для учителя и ученика.

Приложение основано на двух методических принципах: коммуникативности и ситуативности. Ученик получает разнообразные возможности для практики разговорной речи, и эта практика осуществляется в условиях, максимально приближенных к реальной коммуникативной ситуации. Всего разработано тринадцать сценариев в четырёх различных локациях: спортивный зал, кафе быстрого питания, галерея и отель. Помимо комплекса обстановки и предметов, коммуникативные ситуации включают ролевые, социально-статусные, деятельностей и нравственные отношения говорящих.

С октября 2020 по апрель 2021 тренажёр участвовал во Всероссийской программе апробации образовательных приложений в виртуальной и дополненной реальности. Было получено 76 заявок от общеобразовательных организаций среднего образования. В тестировании разработки приняли участие более 800 учеников [13].

Обратная связь от участников Программы анализируется в рамках исследования Центра HTИ VR/AR ДВФУ совместно со специалистами Российской Академии Наук.

\section{4 Приложения для изучения лексики}

Существует несколько мобильных приложений для запоминания слов; не так давно к ним добавились подобные приложения с использованием технологии виртуальной реальности. Изучение вокабуляра здесь аналогично физическим словарным карточкам или изучению слов методом стикеров, который многим хорошо известен: на все объекты окружающего пространства (как правило, дом или офис) приклеиваются стикеры с названиями этих объектов (холодильник, зеркало, дверь). Так, надевая шлем для виртуальной реальности и загрузив приложение VR Learn English [16] от ProVR Games, учащийся попадает в виртуальную квартиру с крайне простым, схематичным дизайном. Учащийся двигается по квартире сообразно движению головы, при этом слышит звуковую репрезентацию окружающих объектов и письменные варианты соответствующих слов. Приложение интуитивное, быстро грузится, а примитивная графика не вызывает сильного отторжения, поскольку ее достаточно для реализации главной функции приложения: запоминания слов. С точки зрения методологии может вызвать вопрос эффективности изучения лексики в отрыве от контекста, но возможность обучения без использования родного языка учащегося является очень важным достоинством приложения с точки зрения коммуникативной методики обучения.

Приложение PanoLingo от ITgenerator [17], которое позиционирует себя как первое приложение для изучения языков, в своей задумке 
не отличается от предыдущего: учащийся также находится в обстановке дома, также изучает написание и звучание окружающих объектов. B PanoLingvo добились большей реалистичности, использовав панорамные фото на 360 градусов, но лишили учащегося возможности перемещения в пространстве (имитации перемещения). В программе, кроме дома, есть и другие локации, например, автобус, магазин или офис. Главным недостатком приложения на текущий момент является его недоработанность: начиная с официального сайта, где в текстах встречаются грамматические ошибки, заканчивая серьёзными ошибками в самой программе, которые, например, не дают передвигаться от одного слова к другому в процессе обучения.

Тем не менее можно констатировать, что техники запоминания новых слов в процессе изучения иностранного языка пополнились еще одним инструментом, который доступен с использованием технологии VR. Эффективность таких техник представляет собой перспективное направление для исследований.

Завершая обзор основных специализированных приложений для изучения иностранных языков, нельзя не упомянуть, что некоторые продукты находятся в процессе разработки. Например, ImmerseME от Immerseme Ltd. [18]. Здесь виртуальная реальность дополнит существующие курсы и логично встроится в систему обучения. Планируется, что это будут панорамные видео 360о с записью речи реального актёра, с которым учащийся сможет иметь нечто похожее на диалог. Другая интересная программа, находящаяся в разработке игра в виртуальной реальности для изучения языков Argotian [19]. Создатели игры утверждают, что комбинация средств виртуальной реальности, искусственного интеллекта и WebXR позволит определить уровень владения языком конкретного пользователя и выстроить соответствующую индивидуальную траекторию обучения. Насколько успешной будет реализация этих идей, покажет будущее.

На данный момент отечественный рынок не предлагает готовых продуктов для изучения иностранных языков в виртуальной реальности. Тем не менее достойна упоминания разработка Smart Education [20] от красноярской компании «КТС». Планируется, что учащийся получит возможность отработать коммуникативные навыки, погрузившись в виртуальную среду. На данный момент компанией создано два виртуальных пространства: аэропорт и комната, причём в аэропорту, по утверждению разработчиков, есть возможность поддержать простой диалог с искусственным интеллектом. На данном этапе проект нуждается в серьезных доработках.

Перейдем к приложениям, которые можно использовать для изучения языков, несмотря на то, что эта функция не является основной.

\section{2 Смежные разработки}

Список виртуальных пространств, где можно улучшить свои языковые навыки (в первую очередь, речь идёт об английском языке) гораздо шире, чем список специализированных средств. Здесь можно выделить три группы приложений: виртуальные социальные сети, образовательные платформы и игры. В рамках данного обзора основное внимание уделено первым двум. 


\section{1 Социальные сети}

Виртуальных площадок для общения становится всё больше, например, различные VRChat [21], Altspace [22], VTime [23], Facebook Spaces [24], Recroom [25] и другие. В контексте данного обзора их объединяет одно: возможность языковой практики, а выбор конкретной платформы - вопрос предпочтений самого учащегося.

Рассмотрим, как это работает на примере программы Altspace, которая технически повторяет VRChat, за исключением некоторых технических ограничений, (например, меньшие возможности в выборе аватара). Слоган Altspace можно перевести как «Не виарь в одиночестве», и здесь всё направлено на общение. Есть три основные опции: 1) интерактивные игры с друзьями, 2) виртуальные события, 3) общение с друзьями и незнакомыми пользователями. Познакомиться с кем-то и поддержать разговор на изучаемом языке можно как на виртуальном событии, так и присоединившись к открытой встрече. Участники сообщества, как правило, дружелюбны и сами инициируют общение, видя новичка. Если же какой-то из собеседников ведет себя некорректно или слишком активно, у его аватара можно отключить звук.

В схожей программе VTime сосредоточиться на практике языков легче: красиво отрендеренные пространства (у костра, в переговорной небоскрёба и даже в открытом космосе) рассчитаны на небольшое количество участников и располагают к спокойному общению. На платформе можно как встречаться с друзьями, так и знакомиться с новыми людьми. VTime - это кроссплатформенная социальная сеть, или, как они себя называют, cross-reality social network. Термин «кроссреальность» используется не просто так. Vtime дает три опции для общения: VR, AR (нужен шлем) и экранная версия (шлем не нужен).

Некоторые преподаватели языков уже используют виртуальные социальные пространства для проведения различных разговорных клубов. Думается, что такой формат занятий подходит интровертам: некоторые учащиеся могут чувствовать себя спокойнее и безопаснее, используя аватар. Кроме того, есть студенты, которые официальному учебному классу предпочитают более неформальную и более естественную внеклассную обстановку, в которой им проще расслабиться, а это часто оказывается важным для практики разговорной речи.

\section{2 Образовательные платформы}

Если преподаватель хочет проводить уроки, приближенные к традиционному формату, и использовать на своих занятиях презентации, текстовые материалы, различные объекты, видео и прочее, то виртуальная реальность предлагает специально разработанные образовательные платформы.

EngageVR [16] от Immersive VR Education позволяет собирать аудиторию до шестидесяти человек, используя на занятиях не только стандартные инструменты типа доски, но и различные 3D-объекты, которыми можно манипулировать в виртуальном пространстве (например, увеличить молекулу до гигантских размеров, делая лекцию более живой и наглядной). Кроме того, платформа содержит несколько готовых обучающих уроков, а также серию видео 360 , которые можно встраивать в структуру урока. К сожалению, несмотря на амбициозность проекта и перспективность разработки, платфор- 
ма всё ещё не готова к использованию. Во-первых, каждое учебное пространство загружается очень долго, в среднем 15-20 минут. Это можно было бы объяснить загрузкой всей сцены из сервера, но при закрытии сервера все загруженные данные удаляются. Во-вторых, использование функций программы, таких как манипуляция с объектами, требует дополнительных навыков со стороны преподавателя в силу неинтуитивности интерфейса.

Платформа Rumii [26] от Doghead Simulations, обладая тем же набором функций и инструментов (доска, 3D-объекты, демонстрация экрана, обмен файлами) работает быстрее и является более интуитивной в освоении, хотя отличается более простой графикой. Разработчики позиционируют себя как «иммерсивный офис», делая акцент на том, что Rumii - пространство для встреч, совместной удалённой работы и учёбы. Большим достоинством программы является кроссплатформенность, включая возможность работы на iOS. В случаях, когда учебное заведение не может приглашать на занятия преподавателя-носителя языка, использование Rumii видится наиболее оправданным.

Последняя разработка, стоящая упоминания, - ClassVR [27] от Avantis Systems Ltd. Компания выпускает собственный шлем виртуальной реальности, используемый исключительно для занятий по специально разработанным программам для нескольких школьных дисциплин (история, естественные науки, искусство и культура, основы безопасности и прочее). Такая автономность упрощает внедрение и использование технологии: приобретается набор из восьми или четырех шлемов в специальном портативном ящике для хранения, и набор сразу готов к использованию. Помимо контента для виртуальной, дополненной и смешанной реальностей, материалы к занятиям включают планы уроков, тесты, игры, кейсы и другие задания. Разработчики, кроме того, предлагают бесплатную демонстрацию и обучение учителей. Минусом является высокая стоимость обучающих комплектов и ориентация на школьную программу Великобритании.

\section{Заключение}

Существующие средства изучения иностранных языков в виртуальной реальности представляет собой совокупность разрозненных разработок разной степени качества и завершенности. В отличие от рынка учебников по английскому языку, где есть свои лидеры и каноны, выработанные в ходе многочисленных исследований, в сфере виртуальной реальности таких лидеров нет. Пока только можно говорить о трёх главных направлениях использования VR-технологии для изучения иностранных языков. Это виртуальная реальность как:

1. Автономный инструмент для тренировки того или иного лингвистического навыка или навыков;

2. Компонент комплексной программы;

3. Образовательная среда, альтернативная физическому учебному пространству.

\section{Заключительные комментарии}

Техники и инструменты обучения иностранному языку, предлагаемые в виртуальной реальности, являются ещё одним инструментом, который дополняет существующий набор методических средств. Эф- 
фективность данного инструмента по сравнению с другими способами дистанционного освоения языков (Skype, мобильные приложения, онлайн курсы) нуждается в дополнительных исследованиях.

Технология виртуальной реальности в обучении иностранным языкам может эффективно применяться для решения частных методических задач, таких как отработка элементарных диалогов, запоминание лексики, языковая разговорная практика и т. д. и должна встраиваться в более широкую методологическую систему.

В контексте коммуникативных ситуаций и практики речи, виртуальная реальность пересекается с технологиями искусственного интеллекта и распознавания речи.

Несмотря на то, что доступ к технологиям виртуальной реальности пока не является массовым, в будущем можно с осторожностью прогнозировать новый виток в развитии индустрии онлайн-курсов. VR, эффективно интегрированный в структуру онлайн курса, предоставляет больше возможности для практической отработки изученных материалов. Возможно, такой вектор развития выведет языковые онлайн курсы на качественно новый уровень.

Несмотря на все достоинства и потенциал, следует помнить, что использование технологии виртуальной реальности должно быть разумным и соответствовать принципу дидактической целесообразности, который предполагает интеграцию новых технологий только в тех ситуациях, когда решение учебной задачи будет эффективнее с этой технологией, нежели при помощи традиционных методов [2]. Думается, что следует избегать внедрения «VR ради VR», как бы много надежд на эту технологию ни возлагалось.

\section{Литература}

1. Доброва В. В., Лабзина П. Г. Виртуальная реальность в преподавании иностранных языков // Вестник Самарского государственного технического университета. 2016. № 4. С. 13-20

2. Скакунова В. А. Дидактические принципы создания информационно-коммуникационной среды в обучении английскому языку (на высшей ступени образования) // Лингвистика, лингводидактика, лингвокультурология: актуальные вопросы и перспективы развития. 2019. № 102. C. 263-266.

3. Blyth C. Immersive technologies and language learning // Foreign Language Annals. 2018. № 51 (1). P. 225-232.

4. Bonner E., Reinders H. Augmented and Virtual Reality in The Language Classroom: Practical Ideas // Teaching English with Technology. 2018. № 18 (3). P. 33-53.

5. Mabrook R., Singer J. Virtual Reality, $360^{\circ}$ Video, and Journalism Studies: Conceptual Approaches to Immersive
Technologies // Journalism Studies. 2019. P. 1-17.

6. Nicosia G. Developing an online writing intensive course: will it work for public speaking? // International Journal of Instructional Media. 2005. № 32 (2). P. 163.

7. Peeters D., Dijkstra T. Sustained inhibition of the native language in bilingual language production: A virtual reality approach // Bilingualism: Language and Cognition. 2018. № 21 (5). P. 1035-1061.

8. Rubio F. Assessment of oral proficiency in online language courses: Beyond reinventing the wheel // The Modern Language Journal. 2015. № 99 (2). P. 405-408.

9. Russell D. The Design of Immersive Virtual Learning Environments Utilizing Problem-Based Learning Templates // Computer-Assisted Language Learning: Concepts, Methodologies, Tools, and Applications - IGI Global, 2019. P. 430-449.

10. Scrivner O., Madewell J., Buckley C., Perez N. Best Practices in the Use of 
Augmented and Virtual Reality Technologies for SLA: Design, Implementation, and Feedback // Teaching Language and Teaching Literature in Virtual Environments - Springer, Singapore. 2019. P. 55-72.

11. Slater M. Immersion and the illusion of presence in virtual reality // British journal of psychology. 2018. № 109 (3). P. 431.

12. Zou D., Xie H., Wang F. L. Future trends and research issues of technologyenhanced language learning: A technological perspective // Knowledge Management \& E-Learning: An International Journal. 2018. № 10 (4). P. 426 - 440.

13. Центр НТИ ДВФУ: Официальный сайт. - Владивосток, 2017-2020. - URL: https://edu.vrnti.ru/varvara (дата обращения: 18.05.2021).

14. Официальный сайт MondlyVR. URL: https://www.mondly.com (дата обращения: 18.05.2021).

15. Официальный сайт VirtualSpeech Ltd. URL: https://virtualspeech.com (дата обращения: 18.05.2021).

16. Официальный сайт Varwin. URL: https://varwin.com (дата обращения: 18.05.2021).

17. Официальный сайт ITgenerator. All rights reserved. URL: https://www.itgenerator. com (дата обращения: 18.05.2021).

18. Официальный сайт ImmersemeVR.
URL: https://immerseme.com (дата обращения: 18.05.2021).

19. Официальный сайт Virtro Entertainment Inc. URL: https://www. argolingo.com (дата обращения: 18.05.2021).

20. Официальный сайт Красноярск Техно Сервис. URL: http://ktservice.ru (дата обращения: 18.05.2021).

21. Официальный сайт VRChat Inc. URL: https://hello.vrchat.com (дата обращения: 18.05.2021).

22. Официальный сайт AltspaceVR. URL: https://altvr.com (дата обращения: 18.05.2021).

23. Официальный сайт vTime Holdings Limited. URL: https://vtime.net (дата обращения: 18.05.2021).

24. Официальный сайт Facebook Spaces. URL: https://www.facebook.com/spaces (дата обращения: 18.05.2021).

25. Официальный сайт Rec Room (Junior Mode). URL: https://recroom.com (дата обращения: 18.05.2021).

26. Официальный сайт Doghead Simulations. URL: https://www. dogheadsimulations.com/rumii (дата обращения: 18.05.2021).

27. Официальный сайт Avantis Systems Ltd. URL: https://www.avantiseducation.com (дата обращения: 18.05.2021). 
Julia S. KHUKALENKO,

Ph. D. (in Philology), Research Fellow at the Centre of National Technology Initiative, Far Eastern Federal University (Vladivostok, Russia).

E-mail: khukalenko.ius@dvfu.ru

\title{
Foreign Language Acquisition (ESL) in Virtual Reality: Software Review
}

\begin{abstract}
Analysis of innovative software in virtual reality for learning virtual reality, languages (ESL as an example) has been undertaken. Existing $I T$, software, used in higher education and secondary schools, educational technologies, can be divided into two main groups: specialized applications and practicing platforms. Each software has been described ESL according to its function, application, pedagogical potential and implementation. It has been demonstrated that virtual reality has a potential as an educational tool, however, more methodological work along with scientific research is needed at this stage.
\end{abstract}

For citation: Khukalenko J. S. Foreign Language Acquisition (ESL) in Virtual Reality: Software Review // Oriental Institute Journal. 2021. № 2. P. 118-128. DOI https://doi.org/10.24866/2542-1611/2021-2/118-128

\section{References}

1. Dobrova V. V., Labzina P. G. Virtual'naya real'nost' $\mathrm{V}$ prepodavanii inostrannykh yazykov // Vestnik Samarskogo gosudarstvennogo universiteta. 2016. № 4. S. 13-20

2. Skakunova V. A. Didakticheskie printsipy sozdaniya informatsionnokommunikatsionnoj sredy $\mathrm{v}$ obuchenii anglijskomu yazyku (na vysshej stupeni obrazovaniya) // Lingvistika, lingvodidaktika, lingvokul'turologiya: aktual'nye voprosy i perspektivy razvitiya. 2019. № 102. S. 263-266.

3. Blyth C. Immersive technologies and language learning // Foreign Language Annals. 2018. № 51 (1). P. 225-232.

4. Bonner E., Reinders H. Augmented and Virtual Reality in The Language Classroom: Practical Ideas // Teaching English with Technology. 2018. № 18 (3). P. 33-53.

5. Mabrook R., Singer J. Virtual Reality, $360^{\circ}$ Video, and Journalism Studies: Conceptual Approaches to Immersive Technologies // Journalism Studies. 2019. P. $1-17$.

6. Nicosia G. Developing an online writing intensive course: will it work for public speaking? // International Journal of Instructional Media. 2005. № 32 (2). P. 163.

7. Peeters D., Dijkstra T. Sustained inhibition of the native language in bilingual language production: A virtual reality approach // Bilingualism: Language and Cognition. 2018. № 21 (5). P. 1035-1061.

8. Rubio F. Assessment of oral proficiency in online language courses: Beyond reinventing the wheel // The Modern Language Journal. 2015. № 99 (2). P. 405-408.

9. Russell D. The Design of Immersive Virtual Learning Environments Utilizing Problem-Based Learning Templates // Computer-Assisted Language Learning: Concepts, Methodologies, Tools, and Applications - IGI Global, 2019. P. 430-449.

10. Scrivner O., Madewell J., Buckley C., Perez N. Best Practices in the Use of Augmented and Virtual Reality Technologies for SLA: Design, Implementation, and Feedback // Teaching Language and Teaching Literature in Virtual Environments - Springer, Singapore. 2019. P. 55-72.

11. Slater M. Immersion and the illusion of presence in virtual reality // British journal of 
psychology. 2018. № 109 (3). P. 431.

12. Zou D., Xie H., Wang F. L. Future trends and research issues of technologyenhanced language learning: A technological perspective // Knowledge Management \& E-Learning: An International Journal. 2018. № 10 (4). P. 426 - 440.

13. TSentr NTI DVFU: Ofitsial'nyj sajt. - Vladivostok, 2017-2020. - URL: https:// edu.vrnti.ru/varvara (data obrashheniya: 18.05.2021).

14. Ofitsial'nyj sajt MondlyVR. URL: https://www.mondly.com (data obrashheniya: 18.05.2021).

15. Ofitsial'nyj sajt VirtualSpeech Ltd. URL: https://virtualspeech.com (data obrashheniya: 18.05.2021).

16. Ofitsial'nyj sajt Varwin. URL: https:// varwin.com (data obrashheniya: 18.05.2021).

17. Ofitsial'nyj sajt ITgenerator. All rights reserved. URL: https://www.itgenerator.com (data obrashheniya: 18.05.2021).

18. Ofitsial'nyj sajt ImmersemeVR. URL: https://immerseme.com (data obrashheniya: 18.05.2021).

19. Ofitsial'nyj sajt Virtro Entertainment
Inc. URL: https://www.argolingo.com (data obrashheniya: 18.05.2021).

20. Ofitsial'nyj sajt Krasnoyarsk Tekhno Servis. URL: http://ktservice.ru (data obrashheniya: 18.05.2021).

21. Ofitsial'nyj sajt VRChat Inc. URL: https://hello.vrchat.com (data obrashheniya: 18.05.2021).

22. Ofitsial'nyj sajt AltspaceVR. URL: https://altvr.com (data obrashheniya: 18.05.2021).

23. Ofitsial'nyj sajt vTime Holdings Limited. URL: https://vtime.net (data obrashheniya: 18.05.2021).

24. Ofitsial'nyj sajt Facebook Spaces. URL: https://www.facebook.com/spaces (data obrashheniya: 18.05.2021).

25. Ofitsial'nyj sajt Rec Room (Junior Mode). URL: https://recroom.com (data obrashheniya: 18.05.2021).

26. Ofitsial'nyj sajt Doghead Simulations. URL: https://www.dogheadsimulations.com/ rumii (data obrashheniya: 18.05.2021).

27. Ofitsial'nyj sajt Avantis Systems Ltd. URL: https://www.avantiseducation.com (data obrashheniya: 18.05.2021). 